\title{
How Are LED Illumination Based Multispectral Imaging Systems Influenced by Different Factors?
}

\author{
Raju Shrestha and Jon Yngve Hardeberg \\ The Norwegian Colour and Visual Computing Laboratory, \\ Gjøvik University College, Gjøvik, Norway \\ raju.shrestha@hig.no
}

\begin{abstract}
LED illumination based multispectral imaging (LEDMSI) is one of the promising techniques of fast and effective spectral image acquisition. Several LEDMSI systems and methodologies have been proposed in the literature. A typical LEDMSI system uses a monochrome camera, which captures images of a scene under $n$ different color LED lights, producing an $n$-band spectral image of the scene. RGB camera based LEDMSI systems have been proposed to speed up the acquisition process. However, demosaicing process in these systems affects the spatial accuracy, and in turn influences the quality of resulting spectral images. In this paper, we study how the performance and quality of LEDMSI systems are influenced by different factors. Four major factors: camera type, demosaicing, number of color LEDs and, noise are considered in the study. We carry out simulation experiments using monochrome and RGB camera based LEDMSI systems, under the influence of different amounts of noise and practical constraints on the number of different color LEDs. The experiments confirm the influence of these factors on the performance of a LEDMSI system. We believe that this work would be useful not only in designing LEDMSI systems, but also in developing quality framework(s) for the evaluation of spectral images and spectral imaging systems.
\end{abstract}

Keywords: spectral imaging, light emitting diodes, demosaicing, noise, quality.

\section{Introduction}

LED (Light Emitting Diode) illumination based multispectral imaging (LEDMSI) has received much attention in recent years due to its fast computer controlled switching ability, robustness, and cost effectiveness. Availability of many different color and high intensity LEDs with peak wavelengths spanning the whole visual range and even infrared region has made the construction of more effective multispectral systems possible, and with little effort.

In a typical LEDMSI, a set of $n$ different color LEDs are selected, each color LED is illuminated in a sequence, and a camera captures images under the illuminated LEDs one at a time, thus producing $n$-band multispectral image. Such

A. Elmoataz et al. (Eds.): ICISP 2014, LNCS 8509, pp. 61-71, 2014.

(C) Springer International Publishing Switzerland 2014 
a system modulates the illumination and provides a multispectral light source. LEDMSI has been used in several applications like biometrics [1, medical imaging 2, film scanner [3, and cultural heritage [4,5]. Studies are being carried out in order to analyze and address different issues with LEDMSI. Spectral variability of LEDs with angle and time of usage is one example of such studies, done by Martinez at al. 6]. Shrestha and Hardeberg [7] has proposed a binary tree based LED matrix/panel design method which produces an optimal or suboptimal arrangement of LEDs for equal energy and uniform lighting. These studies contributed in further maturing LEDMSI.

A number of LEDMSI systems have been proposed in the literature. These systems can be classified into two major types, based on the type of camera used: monochrome camera based [3, 4, and RGB camera based [5, 8, 9. The number of LEDs used in a LEDMSI system might be constrained by its availability and other practical factors, and hence different systems use different number of color LEDs. RGB cameras use color filter arrays (CFA) in a mosaicked pattern, and three color values in each pixel are obtained through demosaicing. This introduces some artifacts because of pixel estimation errors, which could affect the performance of an RGB camera based imaging system. Advanced image processing techniques help mitigate these effects to some extent, which works well in color imaging intended for human observers. However, this might still have considerable influence in the performance of a spectral imaging system, which is intended beyond color, to capture physical properties of objects in a scene, in the form of spectral reflectances. Furthermore, noise also plays an important role in the performance of these imaging systems. In this paper, we study the influence of all these factors in the performance and the quality of LEDMSI systems.

In the next section, we discuss a general concept of LED illumination based multispectral imaging systems, and present the major factors influencing the performance of these systems. In Section 3. we present the simulation experiments performed and the results obtained. We finally conclude the paper in Section 5]

\section{LED Illumination based Multispectral Imaging, and Influencing Factors}

A typical LED illumination based multispectral imaging system comprises of a camera, LED panel(s), and a computer (or any microprocessor based controller), as illustrated in Figure 1. A LED panel is built with a number of different color LEDs. It could be designed in order to make the resulting light as uniform as possible [7. Alternatively, light could be channeled through an optical fiber or an integrating sphere. Any non-uniformity in the illumination can be corrected using flat-field correction during the camera calibration [9, 10. In this research, we assume that the non-uniformity of the illumination is somehow taken into account to have a uniform illumination throughout the whole scene. The controller turns a color LED or LED combination illuminating a scene, and an image is 




Fig. 1. Illustration of a LED illumination based multispectral imaging system

captured by the camera in sync with the light. This is done in a sequence with different color LEDs or LED combinations, turning one at a time. The camera could be monochrome or RGB, based on whether it is a monochrome based or an RGB based LEDMSI system.

In a monochrome camera based LEDMSI system (Mono-LEDMSI), only one type of color LED light is lit at a time [3], where as in an RGB based system (RGB-LEDMSI), a combination of more than one type of color LEDs are lit at a time 5. With $n$ shots, Mono-LEDMSI acquires an $n$-band spectral image, while RGB-LEDMSI acquires a $3 \times n$ band spectral image, thus increasing the capture speed by 3 times. Spectral reflectances of the scene is then estimated from these images using an appropriate spectral estimation method from among a wide variety of methods that have been developed. An optimal number and/or combinations of LEDs are selected from a given set of LEDs. A straightforward method of LEDs selection would be to do exhaustive search under a given criteria, for instance the minimum spectral estimation errors. In the case of an RGB based system, optimal LED combinations can be selected such that each LED in a 3-LED combination splits one of the three spectral sensitivities of the camera in a different region so as to allow the system to capture an image effectively in different spectral bands (wavelength intervals) [5].

The performance and the quality of a LEDMSI is influenced by various factors involved in the design of the system as well as processing involved in it. We introduce here the four major factors that has been studied here in this paper:

Camera type: An RGB camera based system is 3 times faster than a monochrome based system as it can acquire 3-bands image in a single shot. However, in terms of quality the later might be superior, since an RGB camera based system introduces some errors from demosaicing.

Demosaicing algorithm: In an RGB-LEDMSI system, a demosaicing algorithm estimates missing color values in each pixel based on values in other pixels, and 
by doing so it introduces some errors. These errors depend on how accurate a demosaicing algorithm can estimate the pixel values, and hence it in turn influences the quality of acquired spectral images. A number of demosaicing techniques have been proposed in the literature [11 14. An alternative to demosaicing would be to consider $2 \times 2$ pixel as one pixel, and obtain down-sampled spectral images of half the spatial resolution. When a high resolution camera is used, this could be a good compromise, which might be good enough in certain applications.

Noise: It is an unwanted but unavoidable part of digital imaging, since every digital devices introduces different types of noise. We expect poorer quality output with higher noise. However, the influence of noise might be different in different types of LEDMSI systems.

Number of LEDs: The number of LEDs to be used might be constrained by different practical factors, for instance availability, application constraints etc. It might seem logical to think that more the number of LEDs that uniformly cover the EM spectrum, better would be the quality of spectral images captured with it. However, it would be at the cost of speed, and also cost and complexity of the system. Furthermore, with more channels there might be more influence of noise.

\section{$3 \quad$ Experimental Setup}

Simulation experiments are performed in order to study the influence of all the four factors, in the performance of different types of LEDMSI systems. In this section, we first discuss the experimental setup used, and then present the experiments and the results obtained.

LEDMSI systems: Experiments are performed using a number of simulated LEDMSI systems, having different number of LEDs. Spectral sensitivities of a DVC-16000M camera (Figure 2(a) for a monochrome based system, and of a Nikon D600 camera (Figure 2(b) for an RGB based LEDMSI system are used. Two sets of LED lights are used. The first set consists of 19 LEDs from the market, as used by Shrestha and Hardeberg [3]. The second set of LEDs consists of 6 LEDs available in a JUST Normlicht LED ColorControl light booth. The later can be considered as a constrained case which has a limited number of LEDs. Figures 3(a) and 3(b) show relative spectral power distributions of the two sets of LEDs respectively. We use a convention ' $n$-band Mono|RGB-1|2' hereafter, to refer to a monochrome (Mono) or a RGB based LEDMSI system, built with LEDs selected from the LED set 1 or 2 .

Demosaicing algorithms: In order to study the influence of demosaicing algorithms on RGB-LEDMSI systems, we test the systems with five different demosaicing methods: bilinear interpolation [11, adaptive homogeneity-directed demosaicing (AHD) [12, spatially adaptive interpolation based on local polynomial approximation (LPA) and intersection of confidence intervals (ICI) rule 


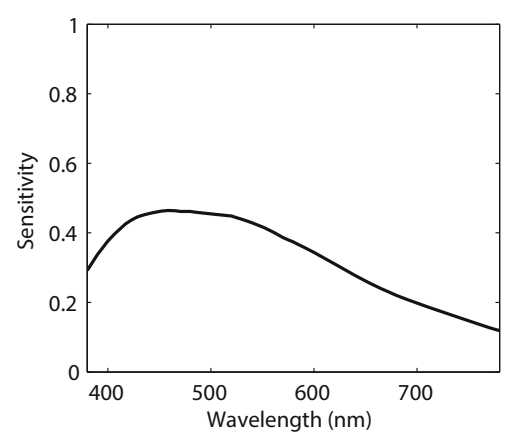

(a) DVC-16000M monochrome camera.

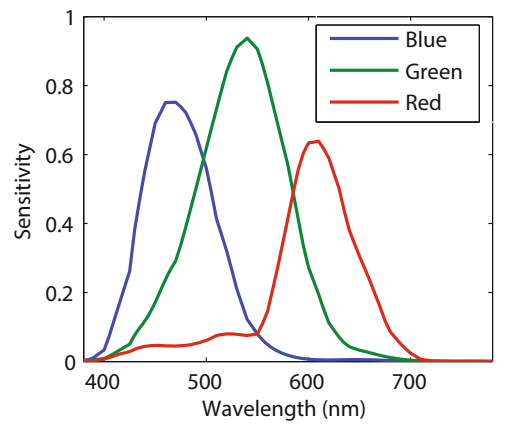

(b) Nikon D600 RGB camera.

Fig. 2. Spectral sensitivities of the cameras used

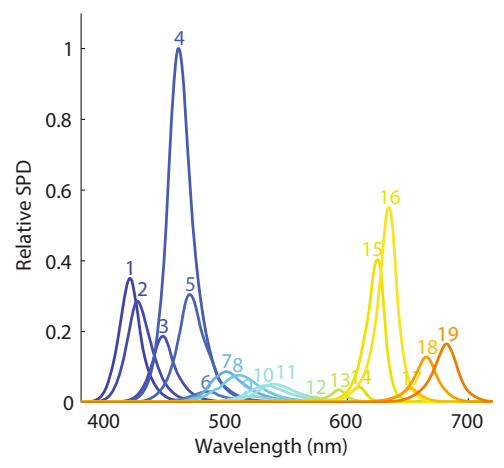

(a) LED set 1:19 LEDs used from Shrestha and Hardeberg [3]

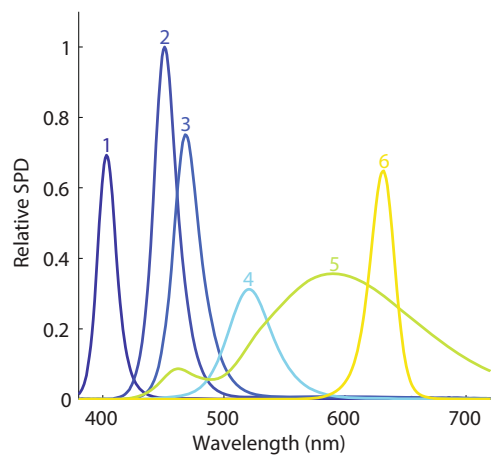

(b) LED set 2: 6 LEDs from JUST Normlicht LED ColorControl light booth.

Fig. 3. Spectral power distributions (SPDs) of LEDs in the two sets of LEDs

(LPA-ICI) [13, and demosaicing based on alternating projections (AP) 14. Down-sampled versions, and ideal systems having three sensors which do not require interpolation are also used, for comparing results.

Test and training targets: In order to evaluate the LEDMSI systems under the study, four hyperspectral images from the University of Eastern Finland's spectral image database [15] have been used to acquire simulated camera images. Figure 4 shows the RGB images rendered from the hyperspectral images.

Surface reflectances of the 240 patches of the Macbeth Color Checker DC (MCCDC) are used for training, and also for testing in the optimal selection of LEDs. Sixty two patches of the MCCDC have been used as the training data set; and one hundred and twenty-two patches remained after omitting the outer surrounding achromatic patches, multiple white patches at the center, and the glossy patches in the S-column of the DC chart, have been used as the test 


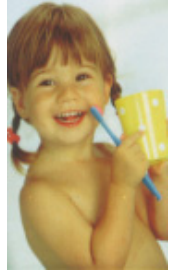

(a) Image 1



(b) Image 2

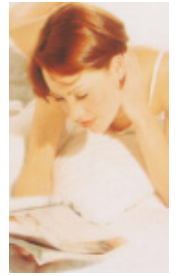

(c) Image 3

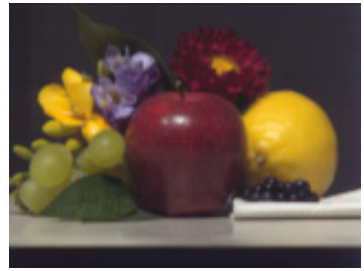

(d) Image 4

Fig. 4. RGB images rendered from the four hyperspectral images from the University of Eastern Finland's spectral image database [15]

data set in the spectral reflectance estimation. The training patches have been selected using the most significant target patches selection method proposed by Hardeberg et al. [16]. The Wiener estimation method [17] is used to reconstruct spectral reflectances of a scene from the camera responses.

Noise: Barnard et al. [18] suggested a realistic level of random shot noise of up to $2 \%$ in a trichromatic camera, under a normal lighting condition. The amount of noise increases when the intensity of the light is low. We therefore investigate the influence of noise on different systems by introducing different amounts (0 to $20 \%$ ) of random Gaussian noise. 16-bit quantization noise is also introduced in order to make the simulated systems more realistic.

Evaluation metric: A number of methods and metrics have been proposed for the evaluation of the performance and the quality of spectral images and spectral imaging systems. In this work, we use the most commonly used spectral metric, the root mean square (RMS) error (RMSE). The concept and methodologies presented in this paper are equally applicable for other metrics also.

\section{Experimental Results and Discussion}

The whole experiment has been divided into four parts: selection of LEDs, influence of demosaicing and noise, and performance of Mono-LEDMSI and RGBLEDMSI systems with different bands.

Selection of LEDs: In this experiment, we select optimal LEDs and LED combinations for the different LEDMSI systems, from a give set of LEDs, through exhaustive search. For an $n$ band Mono-LEDMSI, optimal $n$ different color LEDs are selected which produce the minimum spectral estimation errors (RMSE) from the MCCDC training and the test targets. Similarly for a given number of shots, optimal combinations of 3 LEDs are selected for the RGB-LEDMSI systems. LED selection is done for the both sets of LEDs (Figure 3). Figure 5 shows the LEDs and the LED combinations selected for the six different LEDMSI systems, and the resulting effective channel sensitivities of these systems. From the figures, we see that the selected LEDs cover the whole visual spectrum reasonably well. 




(a) 6-band Mono-1

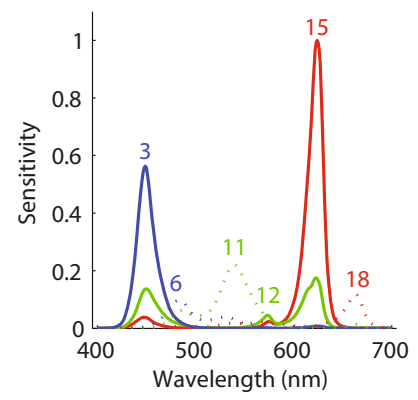

(c) 6-band RGB-1

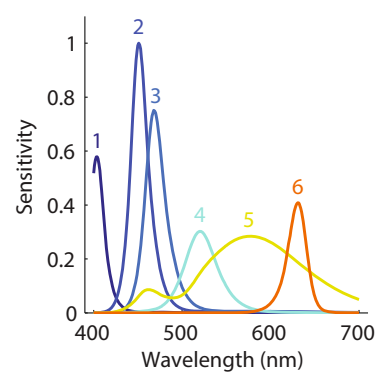

(e) 6-band Mono-2



(b) 9-band Mono-1



(d) 9-band RGB-1

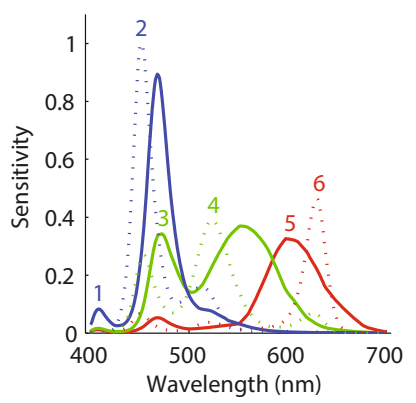

(f) 6-band RGB-2

Fig. 5. LEDs selected for, and the resulting relative channel sensitivities of, the six LEDMSI systems built with monochrome and RGB cameras, and two sets of LEDs. For RGB camera based systems, channel sensitivities in each shot are shown in different line styles.

Influence of demosaicing methods: To study the influence of demosaicing algorithms, a classical 3-band RGB camera and three RGB-LEDMSI systems built with the Nikon D600 camera are simulated to capture the scenes from the four hyperspectral images shown in Figure4. 1\% random Gaussian noise and 16bit quantization noise are introduced in the camera responses. Separate camera images are obtained for the four different demosaicing algorithms introduced in 
Section 3. Spectral reflectances of the scenes are then reconstructed from these camera images. In order to evaluate the performance of a demosaicing algorithm, combined average RMS estimation errors are calculated from all the four images. Figure 6(a) shows the normalized histograms (probability histograms) of the estimation errors produced by the four demosaicing algorithms, along with the corresponding cumulative probability distribution functions (CDF). The figure also shows the results from the down-sampled images as well as from the ideal 3sensor based systems. For the down-sampled case, a reference reflectance image is generated by averaging the four reflectances corresponding to the four pixels in the CFA pattern.

The plots clearly show a significant influence of demosaicing algorithms in the results. As expected, we can see that the results are the best when there is no interpolation done. An interesting result is that the simple bilinear interpolation produces better results with the mean RMSE of 0.013 , compared to the other advanced adaptive demosaicing algorithms. These demosaicing algorithms aim for reducing color artifacts, which works well in color imaging whose goal is to produce visually good (accurate or pleasing color, and less artifacts) images, but this doesn't seem to work well in spectral imaging where we aim for more accurate spectral reflectance estimation. This suggests a need of a special demosaicing technique intended for spectral imaging [19]. Down-sampling also produces better results compared to some of the complex demosaicing algorithms. It is to be noted that we have used low resolution hyperspectral images in our experiments. Much better results can be expected with down-sampling when we use a high resolution camera.

\section{Performance of monochrome and RGB camera based LEDMSI sys-} tems, with different number of bands: In this experiment, we perform experiments to investigate the influence of camera types and the number of LEDs. Simulated 6- and 9-band LEDMSI systems built using the DVC-16000M and the Nikon D600 cameras, and the selected LEDs in the previous experiment are used. Bilinear demosaicing which produces better results among others in the previous experiment is used in the three RGB-LEDMSI systems. Following the same methodology like in the previous experiment, the performance of all the six systems are evaluated using the RMS estimation errors produced by them. Normalized histograms of these estimation errors along with the cumulative probability distribution functions are shown in Figure 6(b)

From the figure, we see that the 9-band Mono-1 system performs the best, producing the mean RMS error of 0.011 with the probability of more than $95 \%$, and the classical 3-band RGB systems performs the worst producing the mean RMS error of 0.048 . The 6 -band Mono-1 system performs slightly worse (mean RMSE of 0.024) but comparable to the 9-band system. The performance of both the 6-band RGB-1 and 6-band RGB-2 systems are similar (mean RMSE of 0.032 and 0.028 respectively), and comparable to the 9-band RGB-1 system (mean RMSE of 0.024). From these results we see that the performance of the 9-band systems are slightly better than 6 -band systems, however the small performance 


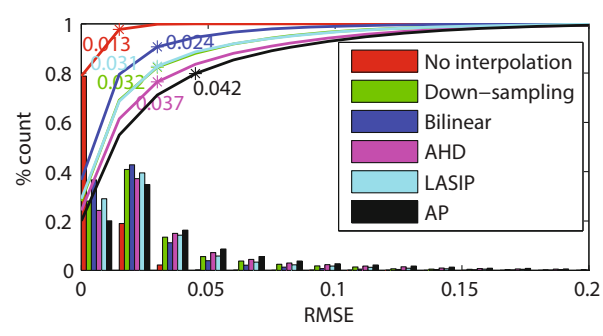

(a) demosaicing methods.

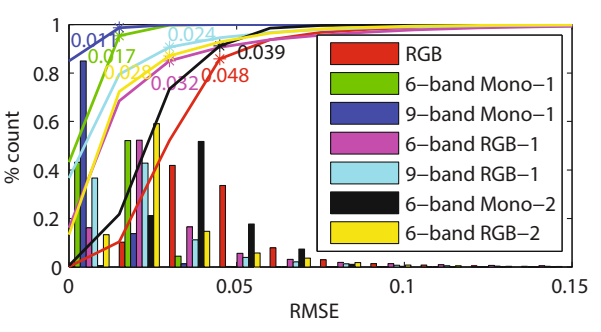

(b) LEDMSI systems.

Fig. 6. Histograms of RMS estimation errors. Cumulative probability distribution functions are also shown, along with the mean error values.

increase may not be considered so significant if we take into account the increase in 3 more bands, lower acquisition speed, and also increase in cost and complexity of the system. One reason behind the small performance improvement is that the influence of noise would be more prominent with the increase in the number of bands. Moreover, increasing the number of LEDs may not always acquire much new information along the spectrum, to produce better results.

An interesting observation is that the 6 -band RGB-2 system seems to perform better than the 6 -band Mono- 2 system. This can be explained if we carefully look at the effective channel sensitivities of the two systems in Figures $5(\mathrm{e})$ and $5(\mathrm{f})$ The six bands in the former system are nicely separated in the visual spectrum, compared to the later system, particularly in the green region. This, probably, allows capture of spectral information better with the 6-band RGB-2 system compared to the Mono-2 system, producing better results.

Influence of noise: In this experiment, we evaluate the influence of noise in the different LEDMSI systems. Random Gaussian noise of different amounts (0 to $20 \%$ ) are introduced to the camera responses, along with the 16-bit quantization noise, and the changes in the RMS estimation errors are noted. Figure 7 shows plots of mean RMS error versus the amount of random Gaussian noise. The plots clearly show the increase in the mean RMS estimation errors with the increase in noise. Furthermore, the influence of noise increases with the increase in the number of bands, which is expected, as more noise would be involved when the number of bands increases. Mean RMS error increases more or less linearly with the noise, in the case of monochrome based systems, whereas the effect is less linear in RGB-LEDMSI systems. This could be due to the effect of demosaicing in RGB camera based systems.

\section{Conclusion}

From the experimental results, we can conclude that the camera type, demosaicing method, number of LEDs and noise have significant influence in a LEDMSI 


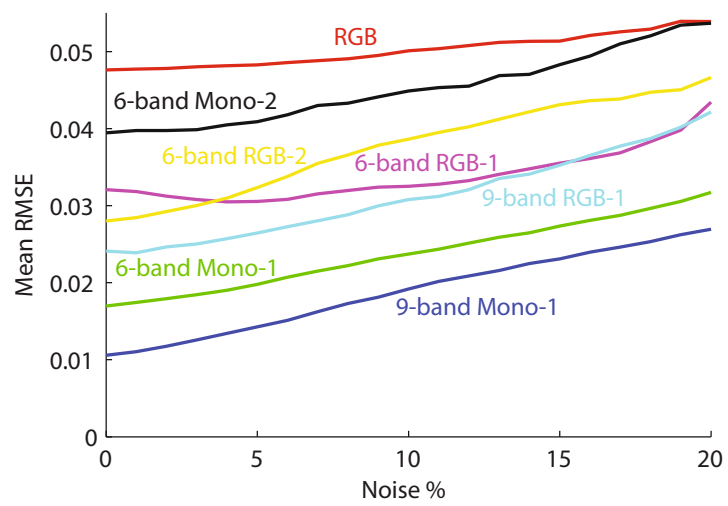

Fig. 7. Plots showing the influence of noise in different LEDMSI systems

system. A monochrome camera based system with wider choice of LEDs along the electromagnetic spectrum, produces better quality outputs. However, RGB camera could be a good choice for speeding up acquisition time by 3 times, possibly at the cost of some reduction in the quality. This is due to the artifacts introduced during demosaicing which affects the quality of the resulting spectral images. With the proper selection of camera and LEDs, an RGB camera based system can provide comparable results, which might be good enough in many applications. Demosaicing algorithms aimed for color imaging might not work well, suggesting for a need of algorithms specifically intended for spectral imaging. The experiments also show that increasing the number of bands might not always produce significantly better performance and quality. LED selection is, therefore, one of the key design aspects of a LEDMSI system.

\section{References}

1. Rowe, R., Uludag, U., Demirkus, M., Parthasaradhi, S., Jain, A.: A multispectral whole-hand biometric authentication system. In: Biometrics Symposium, pp. 1-6 (September 2007)

2. Everdell, N.L., Styles, I.B., Claridge, E., Hebden, J.C., Calcagni, A.S.: Multispectral imaging of the ocular fundus using LED illumination. In: Novel Optical Instrumentation for Biomedical Applications IV, vol. 7371. SPIE Proceedings (2009)

3. Shrestha, R., Hardeberg, J., Boust, C.: LED based multispectral film scanner for accurate color imaging. In: The 8th International Conference on Signal Image Technology and Internet Based Systems (SITIS), pp. 811-817. IEEE Proceedings (November 2012)

4. Christens-Barry, W.A., Boydston, K., France, F.G., Knox, K.T., Easton, J. R.L., Toth, M.B.: Camera system for multispectral imaging of documents. In: Sensors, Cameras, and Systems for Industrial/Scientific Applications X, vol. 7249, pp. 724908-724908-10. SPIE Proceedings (2009) 
5. Shrestha, R., Hardeberg, J.Y.: Multispectral imaging using LED illumination and an RGB camera. In: The 21st Color and Imaging Conference (CIC) on Color Science and Engineering Systems, Technologies, and Applications, pp. 8-13. IS\&T (2013)

6. Martinez, O., Vilaseca, M., Arjona, M., Pizarro, C., Pujol, J.: Use of light-emitting diodes in multispectral systems design: Variability of spectral power distribution according to angle and time of usage. Journal of Imaging Science and Technology 55(5), 50501-1-50501-8 (2011)

7. Shrestha, R., Hardeberg, J.Y.: LED matrix design for multispectral imaging. In: The 12th International AIC Congress, vol. 4, pp. 1317-1320. AIC Proceedings (July 2013)

8. Park, J.I., Lee, M.H., Grossberg, M.D.D., Nayar, S.K.: Multispectral imaging using multiplexed illumination. In: IEEE International Conference on Computer Vision (ICCV), pp. 1-8 (2007)

9. Parmar, M., Lansel, S., Farrell, J.: An LED-based lighting system for acquiring multispectral scenes. In: Digital Photography VIII, vol. 82990, pp. 82990P-82990P-8. SPIE Proceedings (January 2012)

10. Hardeberg, J.Y.: Acquisition and Reproduction of Colour Images: Colorimetric and Multispectral Approaches. Doctoral dissertation, École Nationale Supérieure des Télécommunications de Paris (1999)

11. Longere, P., Zhang, X., Delahunt, P., Brainard, D.: Perceptual assessment of demosaicing algorithm performance. IEEE Proceedings 90(1), 123-132 (2002)

12. Hirakawa, K., Parks, T.: Adaptive homogeneity-directed demosaicing algorithm. IEEE Transactions on Image Processing 14(3), 360-369 (2005)

13. Paliy, D., Katkovnik, V., Bilcu, R., Alenius, S., Egiazarian, K.: Spatially adaptive color filter array interpolation for noiseless and noisy data. International Journal of Imaging Systems and Technology 17(3), 105-122 (2007)

14. Lu, Y., Karzand, M., Vetterli, M.: Demosaicking by alternating projections: Theory and fast one-step implementation. IEEE Transactions on Image Processing 19(8), 2085-2098 (2010)

15. University of Eastern Finland, Spectral Color Research Group: Joensuu spectral image database, https://www.uef.fi/spectral/spectral-image-database (last visit: April 2014)

16. Hardeberg, J.Y., Brettel, H., Schmitt, F.: Spectral characterisation of electronic cameras. In: Electronic Imaging: Processing, Printing, and Publishing in Color, vol. 3409, pp. 100-109. SPIE Proceedings (1998)

17. Haneishi, H., Hasegawa, T., Hosoi, A., Yokoyama, Y., Tsumura, N., Miyake, Y.: System design for accurately estimating the spectral reflectance of art paintings. Applied Optics 39(35), 6621-6632 (2000)

18. Barnard, K., Cardei, V.C., Funt, B.: A comparison of computational color constancy algorithms. I: Methodology and experiments with synthesized data. IEEE Transactions on Image Processing 11(9), 972-984 (2002)

19. Wang, X., Thomas, J.B., Hardeberg, J.: Discrete wavelet transform based multispectral filter array demosaicking. In: IEEE Colour and Visual Computing Symposium, CVCS (September 2013) 